\title{
Lung injury associated with electronic cigarettes
}

\author{
Lisa Saa MS, David Sotello MD
}

\begin{abstract}
As of October $1^{\text {st }}, 2019$, the Centers for Disease Control and Prevention (CDC) has reported 805 known cases of lung injury and 12 deaths associated with electronic cigarettes (e-cigarettes), also known as vapes, across 46 states and one U.S. territory. ${ }^{1}$ The CDC is working to standardize the definition of lung injury associated with e-cigarettes. Currently, it requires patients to have used e-cigarettes during the 90 days prior to presentation, to have bilateral pulmonary infiltrates on imaging, and to have no other identifiable cause for their symptoms. Patients have presented with constitutional, respiratory, and gastrointestinal symptoms, including fever, fatigue, weight loss, shortness of breath, cough, chest pain, vomiting, and diarrhea. ${ }^{1}$

The exact cause of this illness is unknown, but it is believed to have a chemical basis and not an infectious cause. The Federal Drug Administration is working with local agencies to collect samples of the e-cigarette products used to analyze them for a broad range of chemicals. Patients have reported using products with nicotine, tetrahydrocannabinol (THC), or both. The New York State Department of Health has shared preliminary findings of its investigation and found very high levels of vitamin $E$ acetate in almost all of the cannabis-containing samples. ${ }^{2}$ However, this is not yet confirmed as the cause, especially as some patients with the illness have reported not using THC containing products.
\end{abstract}

Acute exogenous lipoid pneumonia has been found in five patients who have used e-cigarettes with THC vaping concentrates or oils that become aerosolized with inhalation. They presented with similar symptoms, including dyspnea, fever, nausea, and

Corresponding author: David Sotello Contact Information: David.sotello@ttuhsc.edu DOI: 10.12746/swrccc.v7i31.607 vomiting; laboratory tests identified no infectious cause. Imaging showed diffuse, basilar predominant infiltrates with a range of ground glass opacities and nodular infiltrates. Bronchoscopy with lavage samples showed neutrophils, lymphocytes, and vacuole-laden macrophages. The patients were diagnosed with acute lipoid pneumonia and successfully treated with corticosteroids. One hypothesis is that the aerosolized oils caused an inflammatory reaction in the airways and alveoli. $^{3}$

Although e-cigarettes have been used as a smoking cessation tool, with a perceived view that they are safer than traditional cigarettes, the data concerning the safety of e-cigarettes have not been well-established. The current cases of lung injury associated with e-cigarettes indicate the need for continued research, analysis of compounds used in e-cigarettes to determine if specific chemicals cause toxicity, and standardized reporting of cases.

Keywords: electronic cigarettes, vaping, lung injury

Submitted: 10/2/2019

\section{REFERENCES}

1. Electronic cigarettes. Centers for Disease Control and Prevention.https://www.cdc.gov/tobacco/basic_information/ e-cigarettes/index.htm. Accessed on 10/01/2019.

2. Get the Facts - Electronic Cigarettes (E-cigarettes) and Similar Vapor Products. New York State Department of Health. https://www.health.ny.gov/prevention/tobacco_control/ campaign/e-cigarettes/ Accessed on 10/01/2019.

3. Davidson K, Brancato A, Heetderks P, et al. Outbreak of electronic-cigarette-associated acute lipoid pneumoniaNorth Carolina, July-August 2019. MMWR Morbidity and Mortality Weekly Report 2019;68:784-786. DOI: http://dx. doi.org/10.15585/mmwr. 